Research Paper

\title{
Daughter Cells and Erythroid Cells Budding from PGCCs and Their Clinicopathological Significances in Colorectal Cancer
}

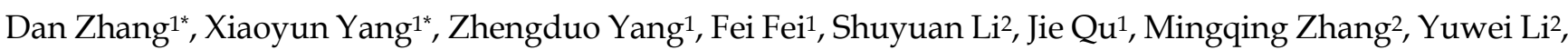 \\ Xipeng Zhang ${ }^{2}$, Shiwu Zhang ${ }^{1}$ \\ 1. Department of Pathology, Tianjin Union Medical Center, Tianjin, 300121, P.R. China. \\ 2. Department of Colorectal Surgery, Tianjin Union Medical Center, Tianjin 300121, P.R.China. \\ *These authors equally contribute to the paper. \\ $\triangle$ Corresponding author: Shiwu Zhang, M.D., Ph.D., Department of Pathology, Tianjin Union Medical Center, Tianjin, 300121, China; Email: \\ zhangshiwu666@aliyun.com. Tel: (86)-13652136865; Fax: (86)-022-87721989. \\ (C) Ivyspring International Publisher. This is an open access article distributed under the terms of the Creative Commons Attribution (CC BY-NC) license \\ (https://creativecommons.org/licenses/by-nc/4.0/). See http://ivyspring.com/terms for full terms and conditions.
}

Received: 2016.07.28; Accepted: 2016.10.17; Published: 2017.02.11

\begin{abstract}
Purpose: We previously reported that polyploid giant cancer cells (PGCCs) induced by cobalt chloride $\left(\mathrm{CoCl}_{2}\right)$ exhibit cancer stem cell properties. Daughter cells generated by PGCCs possess epithelial mesenchymal transition (EMT) phenotype changes and EMT plays an important role in cancer development and progression. This study investigated the characteristics of PGCCs from LoVo and $\mathrm{HCT} 116$ induced by $\mathrm{CoCl}_{2}$ and the clinicopathological significances of PGCCs in colorectal cancer (CRC).

Materials and Methods: Western blotting and immunocytochemical staining were used to compare the expression levels of EMT-related proteins between PGCCs with budding daughter cells and the control cells. In addition, tissue samples were collected from 159 patients with CRC for analysis of PGCCs, vasculogenic mimicry (VM), and single stromal PGCCs with budding, as well as immunohistochemical staining for cathepsin B, vimentin, and hemoglobin $A$.

Results: Single PGCCs induced by $\mathrm{CoCl}_{2}$ formed spheroids in vitro. Poorly differentiated CRCs showed the highest numbers of PGCCs and VM, and expression of cathepsin B. There was greater expression of EMT-related proteins in PGCCs with budding daughter cells than in control cells. The expression of vimentin located in PGCC nuclei. Single stomal PGCCs with budding were detected in $27.45 \%$ of well differentiated, $50 \%$ of moderately differentiated, and $90.20 \%$ of poorly differentiated CRC samples. PGCCs can generate erythroid cells that express delta-hemoglobin to form VM. Erythroid cells generated by PGCCs were positive for hemoglobin A immunocytochemical staining.

Conclusion: PGCCs from LoVo and HCT1 16 treated by $\mathrm{CoCl}_{2}$ exhibited cancer stem cell properties. The number of PGCCs and VM were associated with CRC differentiation and daughter cells budded from PGCCs may promote the lymph node metastasis via expression of EMT-related proteins. PGCCs and their newly generated erythroid cells form VM structures.
\end{abstract}

Key words: Polyploid giant cancer cells, Colorectal cancer, Vasculogenic mimicry, Epithelial-mesenchymal transition, Cancer stem cells.

\section{Introduction}

Colorectal cancer (CRC) is one of the most common malignancies, ranking the third in worldwide cancer incidence [1, 2]. Tumor invasion and metastasis are the main causes of tumor recurrence and patient mortality [3]. Diagnostically, CRC pathologic stage is primarily based upon the invasive depth and metastasis of tumor cells. Twenty percent of all CRCs are metastatic at diagnosis [4]. 
While the overall 5-year survival rate for CRC patients is $64 \%$, this rate drops to $12 \%$ among metastatic CRC patients. Thus, further studies will help clinicians predict CRC pathologic stage, differentiation, metastasis, and prognosis.

Polyploid giant cancer cells (PGCCs) are a special subpopulation of cancer cells that contribute to solid tumor heterogeneity and commonly described histological features of cancer. PGCCs differ from diploid cancer cells in appearance, size, tumorigenic ability, radioresistance, and chemoresistance. PGCCs possess functions of cancer stem cells and thus promote tumor maintenance and recurrence. In paraffin-embedded tissue sections of solid tumors, PGCC formation and function are largely undefined because PGCCs are difficult to isolate and purify. Our previous study showed that PGCC formation can be induced by $\mathrm{CoCl}_{2}$ [5]. PGCCs can generate daughter cells via asymmetric division, resembling mechanisms used in simple eukaryotes and specific cell types in complex eukaryotes. PGCC-derived daughter cells show stronger migratory and invasive capacities than control cells, express less epithelial markers and acquire mesenchymal phenotype [6].

It has recently been recognized that tumor budding serves as an independent prognostic factor and is correlated with lymph node and distant metastasis, recurrence, and prognosis [7]. Nakamura and Kanazawa defined tumor budding as the presence of isolated single cells or small cell clusters (up to five) that are scattered in the stroma at the invasive margin of tumor [8, 9]. Tumor cell morphology at the invasion front is different from morphology in the tumor center [10]. In fact, tumor budding is composed of single stromal PGCCs or daughter cells budded from PGCCs. The budding daughter cells with strong migratory and invasive ability easily metastasize to lymph nodes or distant organs by expressing EMT-related proteins.

Angiogenesis is a vital process for tumor development and progression, and endothelium-dependent vessels (EVs) are the most important transport channel for supplying blood to tumors. Vasculogenic mimicry (VM) is another tumor blood supply pattern adopted by tumor cells and/or basement membranes in the absence of endothelial cells. Sun et al. reported that hypoxia inducible factor-1a (HIF-1a) plays an important role in VM formation [11]. Moreover, our previous studies demonstrated that PGCCs were able to generate erythroid cells that contain fetal hemoglobins, which can strongly bind $\mathrm{O}_{2}$. Daughter cells and erythroid cells generated by PGCCs may form VM structures, and these VM channels can connect EVs to obtain sufficient blood and oxygen to facilitate tumor growth, invasion, and metastasis. In this study, we also investigated the clinicopathological significance of PGCC, VM, cathepsin B expression, and single stromal PGCCs with budding in CRC. We provided evidence confirming that VM and PGCCs are present in human CRC and that PGCC numbers and VM structures are associated with the malignant degree of CRC.

\section{Materials and methods}

\section{Cancer cell lines and culture}

The human colorectal cancer cell lines LoVo and HCT116 were purchased from the American Type Culture Collection (USA) and cultured in RPMI-1640 medium supplemented with $10 \%$ fetal bovine serum (FBS), $100 \mathrm{U} / \mathrm{mL}$ penicillin, and $100 \mathrm{~g} / \mathrm{mL}$ streptomycin.

\section{Generation of PGCCs and spheroid formation}

When the confluence of LoVo and HCT116 cells reached $90 \%$, we treated LoVo with $300 \mu \mathrm{M} \mathrm{CoCl} 2$ (Sigma-Aldrich, St. Louis, MO, USA) for 72 hours and HCT116 with $450 \mu \mathrm{M} \mathrm{CoCl} 2$ for 48 hours. Most regular-sized LoVo and HCT116 cells died following $\mathrm{CoCl}_{2}$ treatment, whereas a few scattered PGCCs survived. Ten to 14 days later, the scattered PGCCs generated daughter cells via asymmetric cell division. After 3-4 times of $\mathrm{CoCl}_{2}$ treatments, there were many spheroids appearing in the flask.

\section{Paraffin embedding of spheroids}

Spheroids from the medium were obtained by centrifugation at $1,000 \mathrm{~g}$ for 5 minutes. The supernatant was removed and the spheroids were incubated in $1 \mathrm{~mL} \mathrm{75 \%}$ ethanol for fixation and $50 \mu \mathrm{L}$ eosin to stain the spheroids. Samples were dehydrated with varying concentrations of ethanol. Finally, the spheroids were embedded in paraffin. All these steps were performed in $1.5 \mathrm{~mL}$ vials.

\section{Hematoxylin and eosin (H\&E) staining}

Four-micrometer sections from formalin-fixed, paraffin-embedded spheroid tissues were deparaffinized, rehydrated, and counterstained with hematoxylin for $1 \mathrm{~min}$ and eosin for $2 \mathrm{~min}$. The sections were then dehydrated and mounted on coverslips.

\section{Immunocytochemical (ICC) staining assays}

ICC staining by using an avidin-biotinperoxidase complex was performed as previously described [12]. PGCCs treated with $\mathrm{CoCl}_{2}$ and control LoVo cells were grown on glass coverslips until $90 \%$ confluence, and the cells were incubated overnight 
with the appropriate primary antibodies at $4^{\circ} \mathrm{C}$ overnight. Finally, the cells were counterstained with hematoxylin and examined under a microscope.

\section{Western blotting}

Western blot analyses were performed as described previously [5, 13]. PGCCs, a mixture of recovered PGCCs $(30 \%)$ with their budding daughter cells $(70 \%)$, and regular cell extracts were lysed. Protein concentrations were determined and proteins were separated on a $10 \%$ sodium dodecyl sulfate (SDS) polyacrylamide gel, and then transferred to polyvinylidene fluoride membranes (Amersham Hybond-P PVDF Membrane; GE Healthcare). The antibodies utilized in this study are listed in Table S1. $\beta$-actin (Sigma-Aldrich) was used as a loading control.

\section{Tissue samples}

Paraffin-embedded CRC tissue samples $(n=159)$ were obtained from the Tumor Tissue Bank of Tianjin Union Medical Center between 2009 and 2013. All patients were histologically diagnosed and no patients were treated prior to surgical tumor resection. Pathologically, 51 CRCs were well differentiated, 56 were moderately differentiated, and 52 were poorly differentiated. This study was approved by the Hospital Review Board and the confidentiality of patient information was maintained.

\section{Immunohistochemistry (IHC) and histochemistry}

IHC staining was performed using the avidin-biotin peroxidase method [5]. The sections were incubated overnight at $4^{\circ} \mathrm{C}$ with the appropriate primary antibody (antibody information is listed in Table S1). In addition, after IHC staining with the CD34, the sections were stained with periodic acid-Schiff (PAS) reagent for $15 \mathrm{~min}$, counterstained with hematoxylin, dehydrated, and mounted. Normal human stomach mucosae were used as the positive control for PAS staining.

\section{Review and scoring of immunostained tissue sections}

Cathepsin B expression was quantified according to the method described by Sun et al. [14]. Specifically, both the signal intensity and the percentage of positive cells were evaluated. For staining intensity, 0 denoted no staining; 1 , weakly positive with faint yellow staining; and 2, strongly positive with brown staining. The number of positive cells was evaluated visually, and fields were stratified as follows: 0 (negative) for no positive cells, 1 (weak) for $<10 \%$ positive cells, 2 (moderate) for $11-30 \%$ positive cells, and 3 (strong) for $>31 \%$ positive cells. The sum (staining index) of the staining intensity and positive cell scores was used to determine the final score.

\section{PGCC identification and counting}

PGCCs were not always uniformly distributed in tumor tissue sections and the hot spot fields of PGCC distribution were frequently observed. In this study, we selected 5 hot spot fields to evaluate at $400 \times$ magnification. The size of PGCC nuclei was measured using a micrometer under H\&E-stained tissue sections according to our previous study that characterized a PGCC as a tumor cell with a nucleus at least three times larger than that of a diploid tumor cell [5].

\section{VM identification and quantification}

To identify VM in human CRCs, tissue sections were double-stained with CD34 and PAS, and blood supply structures were observed microscopically at 400× magnification. Similar to the PGCC count, we selected 5 hot spot fields and counted VMs. Under a microscope, VM wall channels are lined with tumor cells, and red blood cells can be found inside the VM, while inflammatory cells and red cell leakage are not observed around the VM.

\section{Statistical analysis}

The statistical software SPSS 13.0 was used to statistically analyze the data in this study. A $P<0.05$ was considered statistically significant. The Kruskal-Wallis test was used to compare differences in PGCC number, VM number and cathepsin B expression among well, moderately, and poorly differentiated CRCs. The Wilcoxon rank test was performed to examine correlations between PGCC number, VM number, and cathepsin B expression.

\section{Results}

\section{Formation of PGCCs and spheroids in response to $\mathrm{CoCl}_{2}$ treatment}

The control LoVo (Fig. 1A -a) and HCT116 (Fig. $1 \mathrm{~B}-\mathrm{a})$ cells were irregular in shape with small apophyses. After treatment with $\mathrm{CoCl}_{2}$, most diploid cells were killed, and PGCCs could be clearly visualized after removing floating dead cells (Fig. $1 \mathrm{~A}-\mathrm{b}$ and $1 \mathrm{~B}-\mathrm{b})$. After recovery from $\mathrm{CoCl}_{2}$ treatment, individual PGCCs grew larger and generated daughter cells via budding (Fig.1A-c and 1B-c). In addition, individual PGCCs grew into spheroids (Fig. $1 \mathrm{~A}-\mathrm{d}$ and $1 \mathrm{~B}-\mathrm{d})$.

\section{Time-lapse images of budding from single PGCCs}

Asymmetric cell division patterns were observed in LoVo PGCCs. The dynamic processes of single 
PGCCs were recorded over the course of 9 days (Fig. 1C). Nine days after $\mathrm{CoCl}_{2}$ treatment, single PGCCs generated hundreds of daughter cells and there was a clear change in the total number of cells. These results suggest that PGCCs utilize the highly efficient DNA replication and cell division mechanisms to achieve rapid malignant growth.

\section{Number of PGCCs associated with CRC differentiation}

Using the definition of PGCCs established by Zhang et al. [5], our cell size measurements and morphologic observations indicated a significant PGCC presence in CRC tissue sections. Specifically, CRC PGCCs have giant or multiple nuclei. In well differentiated CRCs, PGCCs were not obvious and the nuclear sizes of PGCCs were only three to five times larger than those of diploid cancer cell nuclei (Fig. 1D -a). In moderately (Fig. 1D -b) and poorly differentiated CRCs (Fig. 1D -c), the nuclear sizes of some PGCCs were 10 times greater than the nuclei of diploid cancer cells. Higher number of PGCCs were observed in poorly differentiated CRCs than those observed in well differentiated CRCs $\left(X^{2}=125.217, P=\right.$ 0.000; Table 1). IHC staining data showed that some PGCCs were positive for cathepsin B staining, while diploid cancer cells were negative (Fig. 1D -d). The differences in cathepsin B expression among well, moderately, and poorly differentiated CRCs were statistically significant $\left(X^{2}=29.171, \quad P=0.000\right)$ (Table 1).

Table 1. Association of number of PGCCs, cathepsin B expression and single stromal PGCCs with CRC differentiation

\begin{tabular}{|c|c|c|c|c|c|c|c|c|c|}
\hline CRC & $\mathrm{n}$ & $\begin{array}{l}\text { Number of } \\
\text { PGCCs }\end{array}$ & $x^{2}$ & $P$ & $\begin{array}{l}\text { Staining index of } x^{2} \\
\text { cathepsin B }\end{array}$ & $P$ & $\begin{array}{l}\text { The percentage of tumors } \\
\text { with single stromal PGCCs }\end{array}$ & $x^{2}$ & $P$ \\
\hline Well differentiated & 51 & $4.96 \pm 4.04$ & 125.217 & 0.000 & $0.88 \pm 1.21$ & 0.000 & $27.45 \%(14 / 51)$ & 36.899 & 0.000 \\
\hline $\begin{array}{l}\text { Moderately } \\
\text { differentiated }\end{array}$ & 56 & $14.35 \pm 6.62$ & & & $1.04 \pm 1.36$ & & $50 \%(28 / 56)$ & & \\
\hline Poorly differentiated & 52 & $64.18 \pm 28.62$ & & & $2.51 \pm 1.84$ & & $90.20 \%(46 / 51)$ & & \\
\hline
\end{tabular}

A

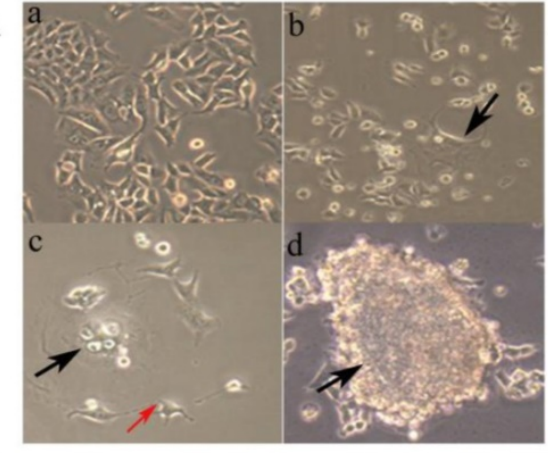

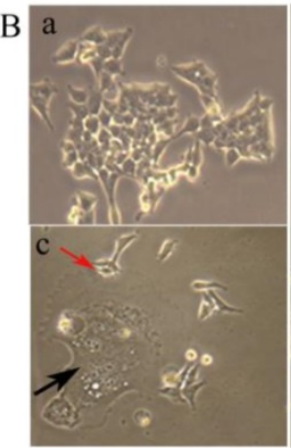
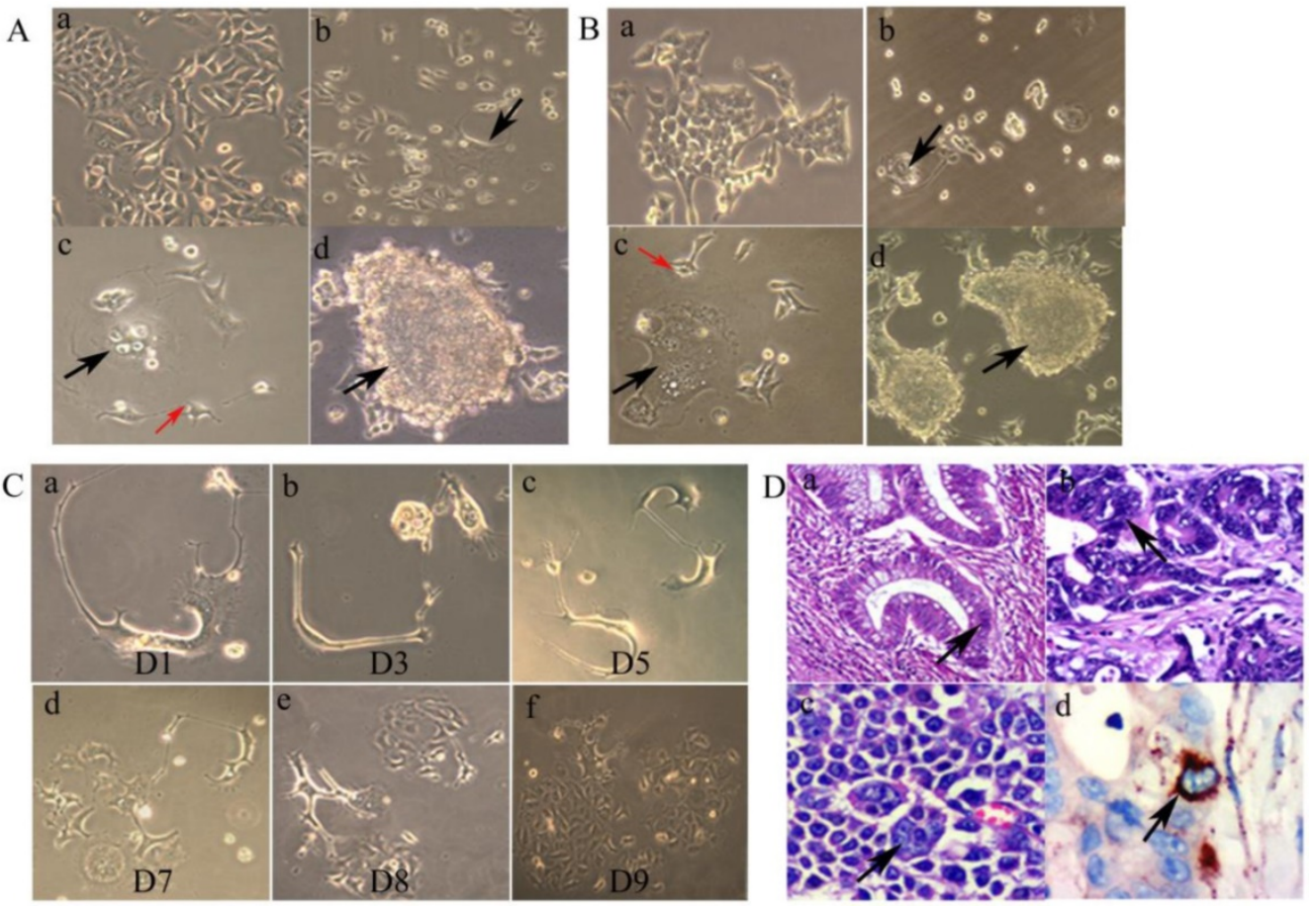

Figure 1. A. Morphologic characteristics of regular LoVo and PGCCs. (a) Morphologic characteristics of regular LoVo cells cultured in complete RPMI-1640 (10x). (b) LoVo PGCCs survived from the treatment of $300 \mu \mathrm{M} \mathrm{CoCl} 2$ for $48 \mathrm{~h}$ (black arrow heads, 10x). (c) Single PGCCs from LoVo (black arrow heads) with their budding daughter cells (red arrow heads, 10x). (d) Formation of cancer spheroids from LoVo PGCCs (black arrow heads, 10x). B. Morphologic characteristics of regular HCT116 and PGCCs. (a) Morphologic characteristics of regular HCT116 cells cultured in complete RPMI-1640 (10x). (b) HCT116 PGCCs survived from the treatment of $300 \mu \mathrm{M} \mathrm{CoCl}_{2}$ for $72 \mathrm{~h}$ (Black arrow heads, 10x). (c) Single PGCCs from HCT116 (black arrow heads) with their budding daughter cells (red arrow heads, 10x). (d) Formation of cancer spheroids from HCT116 PGCCs (black arrow heads, 10x). C. Single LoVo PGCCs generated small daughter cells via budding over a 9-day-period continuous observation (From a to f). D. Presence of PGCCs in CRC tissue specimens and their association with cathepsin B expression. (a) PGCCs in well differentiated CRCs (black arrow heads, H\&E, 20x). (b) PGCCs in moderately differentiated CRCs (black arrow heads, H\&E, 20x). (c) PGCCs in poorly differentiated CRCs (black arrow heads, H\&E, 20x). (d) PGCCs were positive for cathepsin B IHC staining (black arrow heads, IHC, 20×). 

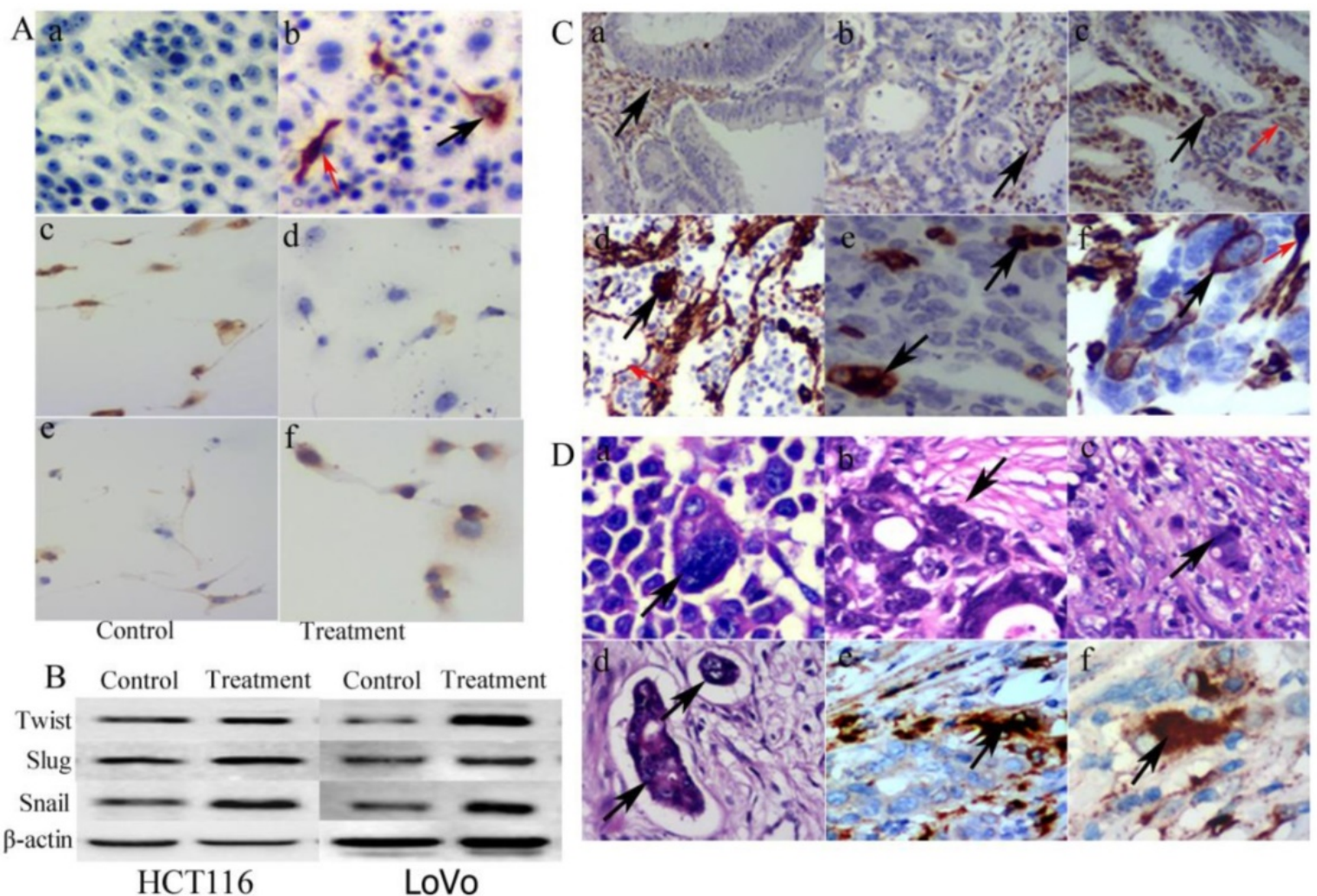

Figure 2. A. ICC staining of vimentin, E-cadherin and N-cadherin. (a) Vimentin staining of control LoVo cells (ICC, 20x). (b) Positive vimentin staining located both in the cytoplasm (black arrow heads) and the nuclei of PGCCs (red arrow heads) (ICC, 20x). (c) Positive E-cadherin staining in control LoVo cells (ICC, 20x). (d) E-cadherin staining in LoVo cells after $\mathrm{CoCl}_{2}$ treatment $(\mathrm{ICC}, 20 \times)$. (e) $\mathrm{N}$-cadherin staining in control LoVo cells (ICC, 20x). (f) Positive N-cadherin staining in LoVo cells after $\mathrm{CoCl}_{2}$ treatment (ICC, 20x). B. Western blot analysis showed the expression of EMT related proteins including Twist, Slug, and Snail expression in LoVo and $\mathrm{HCTl} 16$ before and after $\mathrm{CoCl}_{2}$ treatment. C. Vimentin expression in $\mathrm{CRCs}$ with different differentiation statuses. (a) Stromal cells were positive for vimentin IHC staining in well differentiated CRCs (black arrow heads, IHC, 20x). (b) Stromal cells were positive for vimentin IHC staining in moderately differentiated CRCs (black arrow heads, IHC, 20x). (c) The positive vimentin IHC staining in the nuclei of cancer cells (black arrow heads) and the cytoplasm of stromal cells (red arrow heads) in moderately differentiated CRCs (IHC, 20x). (d) The positive vimentin IHC staining located in the nuclei of PGCC (black arrow heads) and the cytoplasm of stromal cells (red arrow heads) in poorly differentiated CRCs (IHC, 20x). (e) PGCC nuclei were positive for vimentin IHC staining in poorly differentiated CRCs (black arrow heads, IHC, 20x). (f) The positive vimentin IHC staining in both the nuclei (black arrow heads) and cytoplasm (red arrow heads) of PGCCs in poorly differentiated CRCs (IHC, 20x). D. Budding PGCCs were associated with tumor cell invasion. (a) Black arrows indicate daughter cells budded from PGCCs (black arrow heads, H\&E, 20x). (b) PGCCs located at the invasion front (black arrow heads, H\&E, 20x). (c) Single PGCCs located in the stroma (black arrow heads, H\&E, 20x). (d) PGCCs and budded daughter cells formed tumor emboli (black arrow head, H\&E, 20x). (e) PGCCs at the invasion front were positive for cathepsin B IHC staining (black arrow heads, IHC, 20x). (f) Single stromal PGCCs were positive for cathepsin B IHC staining (black arrow heads, IHC, 20x).

\section{Daughter cells budded from LoVo and HCT116 PGCCs exhibit a mesenchymal phenotype}

PGCCs with budding daughter cells and the control LoVo cells were cultured on the coverslips for ICC staining. Results of vimentin ICC staining showed that the control LoVo cells were negative and PGCCs were positive (Fig. 2A $-a$ and $-b$ ). Some positive staining was seen in the cytoplasm and some positive staining was seen in the nuclei of PGCCs (Fig. $2 \mathrm{~A}-\mathrm{b})$. The expression of E-cadherin in the control LoVo cells (Fig. 2A -c) was higher than it in LoVo cells after $\mathrm{CoCl}_{2}$ treatment (Fig. 2A -d) and N-cadherin expression in LoVo cells after $\mathrm{CoCl}_{2}$ treatment (Fig. $2 \mathrm{~A}$-e) was higher than it in the control LoVo cells (Fig. 2A -f). Furthermore, EMT-related proteins were detected in LoVo and HCT116 before and after $\mathrm{CoCl}_{2}$ treatment. Western blot analysis confirmed that there were greater levels of Twist, Slug, and Snail expression in PGCCs and their budding daughter cells compared to control cells (Fig. 2B).

We also performed vimentin IHC staining on the paraffin-embedded samples of CRCs. Well differentiated CRCs were negative for vimentin (Fig. $2 \mathrm{C}-\mathrm{a}$ ), and tumor cells of some moderately and poorly differentiated CRCs were positive (Fig. $2 \mathrm{C}-\mathrm{b}$ to $-\mathrm{f}$ ). Mesenchymal tissue was positive for vimentin IHC staining, confirming the specificity of the vimentin antibody (Fig. 2C -a and -b). Similar to the vimentin ICC staining in vitro, some PGCC nuclei were positive for vimentin (Fig. $2 \mathrm{C}-\mathrm{c},-\mathrm{d}$ and -e) and cytoplasm of some PGCCs was also positive (Fig. 2C-f). Furthermore, PGCCs can produce daughter cells via budding. Figure 2D-a depicts a daughter tumor cell located in the cytoplasm of a PGCC. PGCCs often located at the boundary of tumor infiltration into normal tissue (invasion front; Fig.2D-b) and some 
single PGCCs with budding located in the stroma (single stromal PGCC) (Fig. 2D-c). Daughter cells budded from PGCCs exhibited stronger migratory and invasive ability than the diploid cells. Tumor emboli in blood or lymphatic vessels are also often formed by PGCCs with their budding daughter cells (Fig. 2D-d). Because of the high ability of daughter cells to migrate and invade, PGCCs with budding daughter cells can invade into these vessels to form tumor emboli. Results of ICC data show that PGCCs at the invasion front and single stromal PGCCs were positive for cathepsin B expression (Fig. 2D -e and -f).

\section{Single stomal PGCCs are related to CRC lymph node metastasis}

Next, we evaluated the significance of single stromal PGCCs in CRC biological behavior by carefully observing the distribution of PGCCs and studied the relevance between single stromal PGCCs and lymph node metastasis based on H\&E staining. Single budding stromal PGCCs appeared in $90.20 \%$ $(46 / 51)$ of poorly differentiated CRCs and in only $27.45 \%(14 / 51)$ of well differentiated, and 50\% (28/56) of moderately differentiated CRCs $\left(X^{2}=36.899\right.$, $P=0.000$; Table 1). Lymph node metastasis occurred in $13.73 \%(7 / 51)$ of well differentiated, $32.14 \%(18 / 56)$ of moderately differentiated, and $76.92 \%(40 / 56)$ of poorly differentiated CRCs (Table 2). The predictive values of differentiation levels were $30.61 \%$ (well differentiated tumors), $53.57 \%$ (moderately differentiated tumors), and $84.78 \%$ (poorly differentiated tumors), which showed that single stromal PGCCs have a high predictive value for lymph node metastasis among poorly differentiated CRCs. In contrast, negative predictive values were $91.89 \%, 89.29 \%$, and $83.33 \%$ among well, moderately, and poorly differentiated CRCs, respectively. Poorly differentiated CRCs had the highest metastatic prognostic sensitivity $(97.5 \%)$ and the lowest negative likelihood ratio (0.060), suggesting that single PGCCs with stromal budding had the best predictive value for lymph node metastasis (Table 3).

Table 2. Association of single stromal PGCCs with CRC lymph node metastasis

\begin{tabular}{lllll}
\hline & & \multicolumn{2}{c}{$\begin{array}{l}\text { Lymph node } \\
\text { metastasis }\end{array}$} \\
\cline { 3 - 5 } & & & Yes & No \\
\hline Well differentiated & Single stromal PGCCs & Yes & 4 & 10 \\
& & No & 3 & 34 \\
Moderately & Single stromal PGCC & Yes & 15 & 13 \\
differentiated & & No & 3 & 25 \\
Poorly differentiated & Single stromal PGCC & Yes & 39 & 7 \\
& & No & 1 & 5 \\
\hline
\end{tabular}

Table 3. Sensitivity and Specificity of single stromal PGCCs in detection of CRC lymph node metastasis

\begin{tabular}{llll}
\hline & $\begin{array}{l}\text { Well } \\
\text { differentiated }\end{array}$ & $\begin{array}{l}\text { Moderately } \\
\text { differentiated }\end{array}$ & $\begin{array}{l}\text { Poorly } \\
\text { differentiated }\end{array}$ \\
\hline Sensitivity & $57.14 \%$ & $83.33 \%$ & $97.5 \%$ \\
Specificity & $77.27 \%$ & $65.79 \%$ & $41.67 \%$ \\
False-positive ratio & $22.73 \%$ & $34.21 \%$ & $58.33 \%$ \\
False-negative ratio & $42.86 \%$ & $16.67 \%$ & $2.5 \%$ \\
Positive likelihood ratio & 2.514 & 2.436 & 1.657 \\
Negative likelihood ratio & 0.555 & 0.216 & 0.060 \\
Diagnostic index & 1.344 & 1.491 & 1.392 \\
Youden's index & 0.344 & 0.491 & 0.392 \\
Crude accuracy & $74.51 \%$ & $71.43 \%$ & $84.62 \%$ \\
Positive predictive value & $30.61 \%$ & $53.57 \%$ & $84.78 \%$ \\
Negative predictive value & $91.89 \%$ & $89.29 \%$ & $83.33 \%$ \\
\hline
\end{tabular}

\section{PGCCs generate erythroid cells}

We previously reported that BT-549 PGCCs can generate erythroid cells in vitro and in vivo [15]. For directly examining the nature of spheroids from LoVo and HCT116 cells after $\mathrm{CoCl}_{2}$ treatment, we resuspended the cells from the medium and embedded them in paraffin. Histological examination showed that PGCCs appeared in these spheroids (Fig. 3A -a and -e). Erythroid cells were also clearly generated by LoVo and HCT116 spheroids. Some erythroid cells located in the cytoplasm of PGCCs (Fig. 3A -b and -f) and some located around the PGCCs (Fig. 3A -c and -g). IHC staining analysis demonstrated that the spheroids and erythroid cells were positive for delta-hemoglobin (Fig. 3A -d and -h), while PGCCs with their daughter cells were negative.

\section{PGCCs and their newly generated erythroid cells form VM structures}

PGCCs can produce erythroid cells. Erythroid cells attached to PGCC surfaces (Fig. 3B-a) or located in the cytoplasm (Fig. 3B-b). Erythroid cells that adhered to cancer cell surfaces (Fig. 3B-c) or located in their cytoplasm (Fig. 3B-d) were positive for hemoglobin A staining, whereas the mature red blood cells in the EVS were negative for hemoglobin A (Fig. 3B-c). PGCCs and their generated erythroid cells can form VM structures (Fig. 3B-e).

Typical VM channels consist of tumor cells and red blood cells, and a basement membrane may or may not be present. No endothelial cells were found in the VM inner wall. Moreover, no necrosis or inflammatory cells were observed around VM structures. A PAS-positive basement membrane was not a necessary indication for VM formation. Figure 3C-a showed that VM channels were formed in poorly differentiated CRC, which PAS histochemical and CD34 immunochemical double-staining confirmed that VM channels were negative for PAS and CD34 
staining, while endothelium-dependent vessels were positive for both PAS and CD34 staining (Fig. 3C-b). However, in some VM channels, there was a PAS-positive basement membrane between erythrocytes and tumor cells (Fig. 3C-c). Results of immunochemical and histochemical double-staining confirmed that VM channels were positive for PAS staining (Fig. 3C-d).

To verify the correlation between VM and CRC differentiation, we counted the number of VM structures in CRC tissue samples. Similar to the number of PGCCs, poorly differentiated CRCs had the highest VM channel number, and well differentiated CRCs had the lowest $\left(X^{2}=103.257\right.$, $P=0.000$; Table 4). Statistical analyses showed that PGCC number was positively associated with VM number $\quad\left(\mathrm{r}_{\mathrm{s}}=0.817, \quad P=0.000\right)$ and cathepsin $\mathrm{B}$ expression $\left(r_{s}=0.378, P=0.000\right)$, while VM number was also positively associated with cathepsin $B$ expression $\left(r_{s}=0.390, \quad P=0.000 ; \quad F i g .3 D\right)$. Furthermore, VM distribution was similar to PGCC distribution; VMs were frequently found at the invasion front, which might provide sufficient blood supply for tumor cells to invade adjacent normal tissues.

Table 4. Association of VMs with CRC differentiation

\begin{tabular}{lllll}
\hline CRC & $\mathrm{n}$ & Number of VMs & $\chi^{2}$ & $P$ \\
\hline Well differentiated & 51 & $0.90 \pm 1.03$ & 103.257 & 0.000 \\
Moderately differentiated & 56 & $2.98 \pm 1.72$ & & \\
Poorly differentiated & 52 & $7.80 \pm 5.37$ & & \\
\hline
\end{tabular}

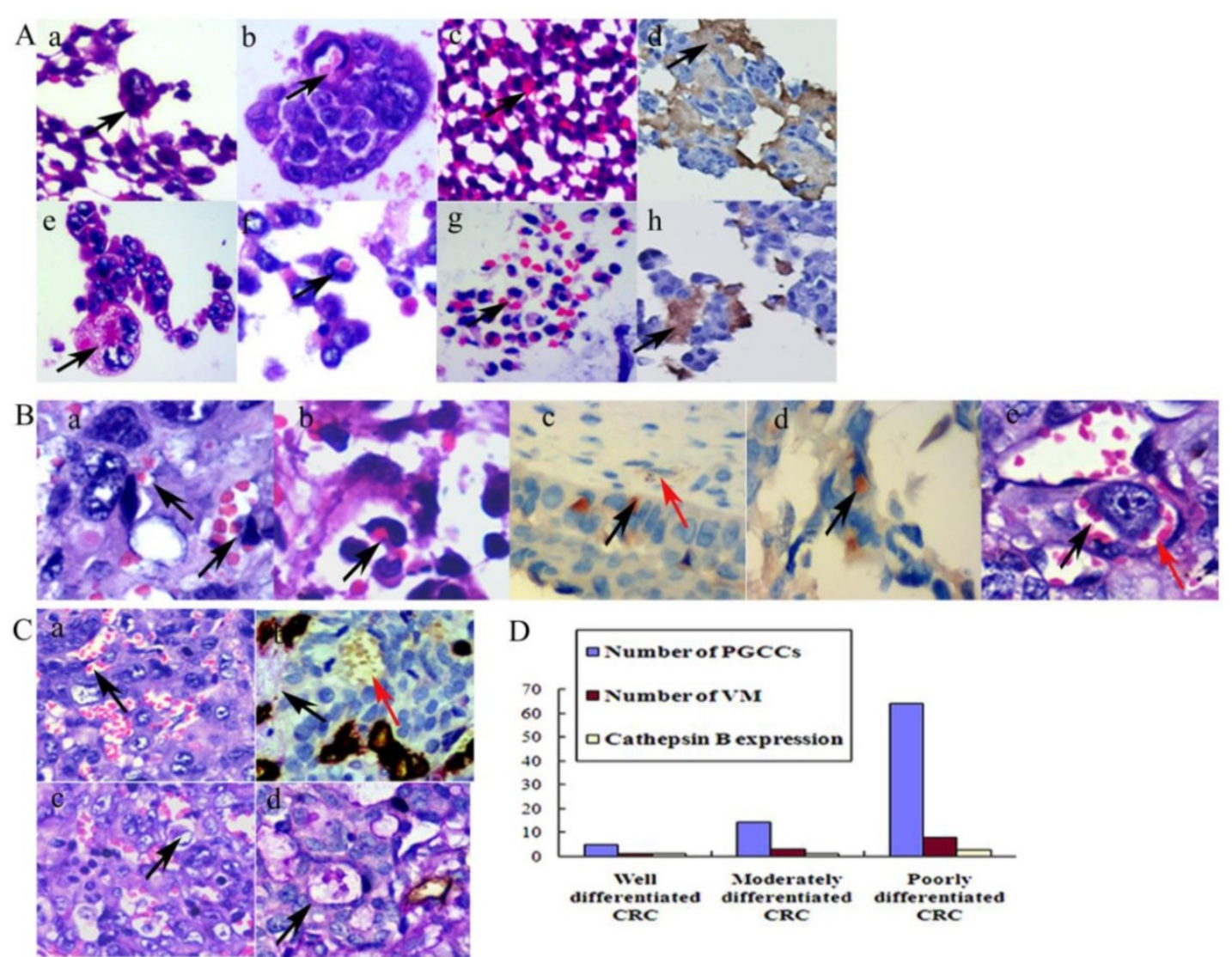

Figure 3. A. Generation of erythroid cells by PGCCs from LoVo and HCT116 cancer cell lines. (a) PGCCs from LoVo were observed in paraffin-embedded spheroids (black arrow heads, H\&E, 20x). (b) Erythroid cells appeared in the cytoplasm of LoVo PGCCs (black arrow heads, H\&E, 20x). (c) Erythroid cells generated from LoVo cell block after $\mathrm{CoCl}_{2}$ treatment (black arrow heads, H\&E, 20x). (d) Erythroid cells in (c) were positive for anti-delta hemoglobin (Black arrow heads, IHC 20x). (e) PGCCs from HCT116 were observed in paraffin-embedded spheroids (black arrow heads, H\&E, 20x). (f) Erythroid cells appeared in HCT116 PGCCs cytoplasm (black arrow heads, H\&E, 20x). (g) Erythroid cells generated from HCTl 16 cell block after $\mathrm{CoCl}_{2}$ treatment (black arrow heads, $\mathrm{H} \& \mathrm{E}, 20 \times$ ). (h) Erythroid cells in (g) were positive for anti-delta hemoglobin (Black arrow heads, IHC, 20x). B. PGCCs produced daughter tumor cells and erythroid cells to form VM. (a) Erythroid cells (black arrow heads) adhere to PGCC surfaces (H\&E, 20x). (b) Erythroid cells located in the cytoplasm of PGCCs (black arrow heads, H\&E, 20x). (c) Erythroid cells adhered to PGCC surfaces were positive for hemoglobin A (black arrow heads) and red blood cells in EVs (red arrow heads) were negative for hemoglobin A (IHC, 20x). (d) Erythroid cells in the cytoplasm of PGCCs were positive for hemoglobin A (black arrow heads, IHC, 20x). (e) Erythroid cells budding from PGCCs (black arrow heads) and tumor cells formed VM (red arrow heads) (H\&E, 20x). C. Presence of VM in CRCs. (a) VM without a PAS-positive basement membrane (black arrowheads, H\&E, 20x). (b) VM without a basement membrane (red arrow heads) and EVs (black arrow heads) that were stained with both CD34 and PAS (double-staining, 20x). (c) VM structure with a PAS-positive basement membrane (black arrow heads, H\&E, 20x). (d) VM with a basement membrane was positive for PAS staining and negative for CD34 staining (black arrow heads, double staining, 20x). D. The relationship between tumor differentiation and cathepsin $\mathrm{B}$ expression levels and the number of PGCCs and VM in CRCs. 


\section{Discussion}

Tumor cell invasion and metastasis are key factors for tumor recurrence and patient mortality. To date, clinical CRC staging is mainly based on tumor cell invasive depth and presence of metastasis. In the study, 159 CRC cases were categorized into three groups: well differentiated, moderately differentiated, and poorly differentiated. We provided evidences showing the presence of PGCCs and VM in human CRC samples, and the numbers of PGCC and VM associated with tumor differentiation. PGCCs generated erythroid cells that can help form VM channels. Single stromal PGCCs with budding were closely associated with tumor lymph node metastasis. The nuclear features of PGCCs are some of the most commonly described histopathological features of solid tumors, and typically became more prominent as the pathologic grade and disease stage increased [16]. In this study, we compared human CRC differentiation status and grade to PGCC numbers. More PGCCs were detected in poorly differentiated tumors than in well differentiated ones. Most PGCCs located around necrotic tissue and in the hypoxic boundary between normal and tumor tissue.

Hypoxia can promote self-renewal of cancer stem cells and a stem cell-like phenotype in many kinds of malignant tumors [17]. We previously reported that PGCCs induced by $\mathrm{CoCl}_{2}$ had cancer stem cell properties and generated daughter cells via asymmetric division [5]. Daughter cells generated by PGCCs possessed a mesenchymal phenotype and exhibited increased migration and invasive capability, which led to single PGCCs with budding daughter cells in the stroma or tumor emboli. Patients whose tumor tissue contained single stromal PGCCs were more likely to have lymph node metastasis than patients without single stromal PGCCs.

EMT facilitates the generation of cancer stem cells and their migratory and invasive properties [18]. In contrast to control cells, daughter cells budded from PGCCs acquired a mesenchymal phenotype and expressed EMT-related proteins including Twist, Slug, Snail, and vimentin. Notably, vimentin was located in the nucleus of PGCCs. In recent years, vimentin has been identified in the cytoplasm of primary cancers [19]. Currently, little is known about nuclear vimentin in clinical tumor tissues. Mergui et al. reported that vimentin regulated p21 transcription and was expressed in nuclei of human neuroblastoma cell lines [20]. Nuclear translocation of vimentin plays an important role in tumor progression and metastasis [21]. However, the molecular mechanisms underlying vimentin nuclear translocation have not been completely clarified. EMT-related proteins including E-cadherin and $\mathrm{N}$-cadherin are widely used in invasion and metastasis cancer research [22]. It is reported that many cancer cells exhibit decreased expression of E-cadherin and increased expression of $\mathrm{N}$-cadherin, which are significantly correlated with the malignant degree of cancers and the metastasis of cancers to the lymph nodes. These alterations are in agreement with the EMT phenotype reported in breast cancer and esophageal squamous cell carcinoma [23]. Furthermore, our current data showed that PGCCs expressed cathepsin B, whereas diploid cancer cells were negative for this protein. Cathepsin B belongs to a family of peptidases, or proteases [24]. This protein is a lysosomal cysteine protease composed of a dimer of disulfide-linked heavy and light chains produced from a single protein precursor. Overexpression of cathepsin B is associated with esophageal adenocarcinoma and other cancers [25]. By using isobaric tags for relative and absolute quantitative proteomic analysis, we found that cathepsin B was markedly elevated in PGCCs compared to diploid tumor cells in the absence of treatment.

In addition to inducing PGCC formation, hypoxia also plays an important role in VM formation [26]. Hypoxia stimulates growth of new blood vessels [27]. VM formation might be a complementary means for tumor cells to acquire adequate oxygen supply and metabolites within a hypoxic environment [28]. Our previous studies showed that melanoma cells in a hypoxic microenvironment had increased HIF-1a expression and that hypoxia can activate some invasion- and metastasis-associated tumor genes, enabling the cancer cells to become more invasive and form VM structures [11]. Our current study also showed that VM was associated with degree of tumor differentiation. VM structures were found more frequently in poorly differentiated CRCs. Sood et al. showed that the molecular profile of aggressive cutaneous and uveal melanoma cells in the presence of VM was similar to that of pluripotent, embryonic-like stem cells. This led us to hypothesize that that tumor cells composed of VM channels can secrete some factor(s) that may promote penetration and metastasis.

Our previous study demonstrated that PGCCs from tumor cell lines and human serous ovarian carcinomas induced by $\mathrm{CoCl}_{2}$ are able to generate erythroid cells. Furthermore, it has been reported that human embryonic stem cells and induced pluripotent stem cells can also generate erythrocytes in vitro [29]. Szabo et al. demonstrated the generation of multilineage blood progenitors from human dermal fibroblasts [30]. Because tumor cells can generate erythroid cells, tumor cells and their newly generated 
erythroid cells can form VM structures during tumor development and progression. It is notable that erythroid cells generated from PGCCs express embryonic and fetal hemoglobin, which have stronger affinity for oxygen than adult hemoglobin does, enabling tumor cells to survive in hypoxic conditions. The generation of erythroid cells from tumor cells provides a rational explanation for how normal and cancer cells can obtain oxygen under hypoxic conditions with or without angiogenesis.

\section{Conclusion}

Our data demonstrate that PGCCs induced by $\mathrm{CoCl}_{2}$ can generate daughter cells with strong migratory and invasive ability by expressing EMT-related proteins. Daughter tumor cells and embryonic erythroid cells generated by PGCCs can form VM to supply tumor cells with oxygen and nutrient supply. Single stromal PGCCs with budding are associated with lymph node metastasis in CRCs. Further studies on the molecular mechanisms by which single budding PGCCs invade into the stroma may shed light on new therapeutic strategies for tumor lymph node metastasis.

\section{Supplementary Material}

Table S1. http:/ /www.jcancer.org/v08p0469s1.pdf

\section{Abbreviations}

PGCCs, polyploid giant cancer cells; HIF-1a, hypoxia induce factor-1a; IHC staining, immunohistochemical staining; VM, vasculogenic mimicry; EVs, endothelium-dependent vessels; PBS, phosphate-buffered saline; H\&E, hematoxylin and eosin; PAS, periodic acid-Schiff; $\mathrm{CoCl}_{2}$, cobalt chloride; CRC, colorectal cancer.

\section{Acknowledgements}

This work was supported in part by grants from the National Science Foundation of China (81472729, 81672426), the Foundation of Tianjin Health Bureau (2014KY29, 2015KY28， 15KG112, 2015KZ060), the Foundation of committee on science and technology of Tianjin (13JCYBJC42700) and the Foundation of Ministry of Education of China (2014-1685).

\section{Conflicts of Interest}

The authors declare no conflict of interest.

\section{References}

1. Herszenyi L, Tulassay Z. Epidemiology of gastrointestinal and liver tumors. European review for medical and pharmacological sciences. 2010; 14: 249-58.

2. Siegel R, Naishadham D, Jemal A. Cancer statistics, 2013. CA: a cancer journal for clinicians. 2013; 63: 11-30.

3. Khan N, Mukhtar H. Cancer and metastasis: prevention and treatment by green tea. Cancer metastasis reviews. 2010; 29: 435-45.
4. Yoon SS, Tanabe KK. Surgical treatment and other regional treatments for colorectal cancer liver metastases. The oncologist. 1999; 4: 197-208.

5. Zhang S, Mercado-Uribe I, Xing Z, Sun B, Kuang J, Liu J. Generation of cancer stem-like cells through the formation of polyploid giant cancer cells. Oncogene. 2014; 33: 116-28.

6. Zhang S, Mercado-Uribe I, Hanash S, Liu J. iTRAQ-based proteomic analysis of polyploid giant cancer cells and budding progeny cells reveals several distinct pathways for ovarian cancer development. PloS one. 2013; 8: e80120.

7. Prall F. Tumour budding in colorectal carcinoma. Histopathology. 2007; 50: 151-62.

8. Kanazawa H, Mitomi H, Nishiyama Y, Kishimoto I, Fukui N, Nakamura T, et al. Tumour budding at invasive margins and outcome in colorectal cancer. Colorectal disease : the official journal of the Association of Coloproctology of Great Britain and Ireland. 2008; 10: 41-7.

9. Nakamura T, Mitomi H, Kanazawa H, Ohkura Y, Watanabe M. Tumor budding as an index to identify high-risk patients with stage II colon cancer. Diseases of the colon and rectum. 2008; 51: 568-72.

10. Choi HJ, Park KJ, Shin JS, Roh MS, Kwon HC, Lee HS. Tumor budding as a prognostic marker in stage-III rectal carcinoma. International journal of colorectal disease. 2007; 22: 863-8.

11. Sun B, Zhang D, Zhang S, Zhang W, Guo H, Zhao X. Hypoxia influences vasculogenic mimicry channel formation and tumor invasion-related protein expression in melanoma. Cancer letters. 2007; 249: 188-97.

12. Jia L, Zhang S, Ye Y, Li X, Mercado-Uribe I, Bast RC, Jr, et al. Paclitaxel inhibits ovarian tumor growth by inducing epithelial cancer cells to benign fibroblast-like cells. Cancer letters. 2012; 326: 176-82.

13. Zhang S, Mercado-Uribe I, Liu J. Tumor stroma and differentiated cancer cells can be originated directly from polyploid giant cancer cells induced by paclitaxel. International journal of cancer Journal international du cancer. 2014; 134: 508-18.

14. Sun $B$, Zhang $S$, Zhang $D, L i$, Zhao $X$, Luo $Y$, et al Identification of metastasis-related proteins and their clinical relevance to triple-negative human breast cancer. Clinical cancer research : an official journal of the American Association for Cancer Research. 2008; 14: 7050-9.

15. Zhang S, Mercado-Uribe I, Liu J. Generation of erythroid cells from fibroblasts and cancer cells in vitro and in vivo. Cancer letters. 2013; 333: 205-12.

16. Marusyk A, Almendro V, Polyak K. Intra-tumour heterogeneity: a looking glass for cancer? Nature reviews Cancer. 2012; 12: 323-34.

17. Mohyeldin A, Garzon-Muvdi T, Quinones-Hinojosa A. Oxygen in stem cell biology: a critical component of the stem cell niche. Cell stem cell. 2010; 7: 150-61.

18. Mani SA, Guo W, Liao MJ, Eaton EN, Ayyanan A, Zhou AY, et al. The epithelial-mesenchymal transition generates cells with properties of stem cells. Cell. 2008; 133: 704-15.

19. Iwatsuki M, Mimori K, Fukagawa T, Ishii H, Yokobori T, Sasako M, et al. The clinical significance of vimentin-expressing gastric cancer cells in bone marrow. Annals of surgical oncology. 2010; 17: 2526-33.

20. Mergui X, Puiffe ML, Valteau-Couanet D, Lipinski M, Benard J, Amor-Gueret M. p21Waf1 expression is regulated by nuclear intermediate filament vimentin in neuroblastoma. BMC cancer. 2010; 10: 473.

21. Tiligada E. Nuclear translocation during the cross-talk between cellular stress, cell cycle and anticancer agents. Current medicinal chemistry. 2006; 13: 1317-20.

22. Jin H MS, Sato F, Kudo Y, Akasaka H, Tsutsumi S, Ogasawara H, Miyamoto K, Wajima N, Kawasaki $\mathrm{H}$, Hakamada K, Kijima H. Vimentin expression of esophageal squamous cell carcinoma and its aggressive potential for lymph node metastasis. Biomed Res 2010; 31: 105-12.

23. Pang L, Li Q, Wei C, Zou H, Li S, Cao W, et al. TGF-beta1/Smad signaling pathway regulates epithelial-to-mesenchymal transition in esophageal squamous cell carcinoma: in vitro and clinical analyses of cell lines and nomadic Kazakh patients from northwest Xinjiang, China. PloS one. 2014; 9: e112300.

24. Chan SJ, San Segundo B, McCormick MB, Steiner DF. Nucleotide and predicted amino acid sequences of cloned human and mouse preprocathepsin B cDNAs. Proceedings of the National Academy of Sciences of the United States of America. 1986; 83: 7721-5.

25. Cao L, Taggart RT, Berquin IM, Moin K, Fong D, Sloane BF. Human gastric adenocarcinoma cathepsin $\mathrm{B}$ : isolation and sequencing of full-length cDNAs and polymorphisms of the gene. Gene. 1994; 139: 163-9.

26. Zhang S, Li M, Zhang D, Xu S, Wang X, Liu Z, et al. Hypoxia influences linearly patterned programmed cell necrosis and tumor blood supply patterns formation in melanoma. Laboratory investigation; a journal of technical methods and pathology. 2009; 89: 575-86.

27. Keith B, Simon MC. Hypoxia-inducible factors, stem cells, and cancer. Cell. 2007; 129: 465-72.

28. Folberg R, Arbieva Z, Moses J, Hayee A, Sandal T, Kadkol S, et al. Tumor cell plasticity in uveal melanoma: microenvironment directed dampening of the invasive and metastatic genotype and phenotype accompanies the generation of vasculogenic mimicry patterns. The American journal of pathology. 2006; 169: 1376-89.

29. Tolar J, Park IH, Xia L, Lees CJ, Peacock B, Webber B, et al. Hematopoietic differentiation of induced pluripotent stem cells from patients with mucopolysaccharidosis type I (Hurler syndrome). Blood. 2011; 117: 839-47. 
30. Szabo E, Rampalli S, Risueno RM, Schnerch A, Mitchell R, Fiebig-Comyn A, et al. Direct conversion of human fibroblasts to multilineage blood progenitors. Nature. 2010; 468: 521-6. 